\title{
Synthesis of crosslinked poly(orthosilicate)s based on cyclohexanediol derivatives and their swelling properties
}

\author{
Koksal Karadag, Gulsah Onaran and Hayal Bulbul Sonmez
}

Condensations of tetraethyl orthosilicate (TEOS) with cyclohexanediol derivative monomers at moderately high temperatures resulted in the creation of crosslinked poly(orthosilicate)s. The structural and thermal properties of the crosslinked poly(orthosilicate)s polymers were determined by Fourier transform infrared spectroscopy, solid-state ${ }^{13} \mathrm{C}$ and ${ }^{29} \mathrm{Si}$ crosspolarization magic angle spinning nuclear magnetic resonance, thermogravimetric analysis and differential scanning calorimetry. The synthesized crosslinked polymers were found to be insoluble in common organic solvents, such as tetrahydrofuran, dichloromethane, benzene and acetone, although they displayed swelling abilities in these solvents. The swelling ability of the crosslinked polymers was examined by the swelling test, swelling kinetics and desorption kinetics. The results suggest that crosslinked poly(orthosilicate)s based on cyclohexanediols can find good use as absorbents for organic solvents. Polymer Journal (2010) 42, 706-710; doi:10.1038/pj.2010.62; published online 14 July 2010

Keywords: crosslinked poly(orthosilicate)s; cyclohexanediols; organogel; swelling; TEOS

\section{INTRODUCTION}

Polymer gels are significant in chemical research because they can absorb solvents and swell to dimensions much larger than their original dry size, depending on the chemical structure of the gel. This property renders them highly important in analytical and industrial applications as absorbent materials and separation agents. $^{1-8}$

Gels can be classified based on their origin, constitution and type of crosslinkage. ${ }^{9}$ Depending on the liquid medium in the polymer network, polymer gels can be separated into two classes: hydrophilic gels, otherwise termed hydrogels, and organic gels, also called organogels. A hydrogel is a polymeric material that can swell and absorb a significant amount of water within its structure. Hydrogels have been extensively studied by many research groups, ${ }^{10-15}$ and their response to external environmental factors such as $\mathrm{pH}$, temperature and solvent is of special interest because of their potential use as drug delivery systems, actuators and separation devices. ${ }^{16-22}$ However, relatively little research has appeared in the literature with regard to organogels. Organogels can absorb organic solvent or oil spilled on water. ${ }^{23}$ For this purpose sorbents should have oleophilic or hydrophobic characteristics. As environmental pollution by oil and organic solvent spillage increases, investigations of absorbent gels are garnering greater interest. To be useful as efficient absorbent materials, polymeric gels should have some intrinsic properties such as a fast absorption rate, a high absorption capacity, reusability and a good absorption selectivity. Hydrophobic network polymers, especially alkyl acrylate and aromatic polymers, are used as absorbents of oils or certain organic solvents. ${ }^{24-28}$ Oil or organic solvent absorption is the process by which an organic molecule penetrates into a network and expands it to cause swelling. This swelling is affected by the following three factors: rubber elasticity, affinity to the solution and crosslinking density. ${ }^{29}$

In the literature, poly(1,4-butadiene) gels are prepared by the crosslinking polymerization of 4-ter-butylstyrene and divinylbenzene into unvulcanized butadiene rubber. The effects of the reaction conditions on the equilibrium swelling ratio have been investigated, and the highest oil absorbencies are found in xylene and cyclohexane. $^{30}$

Kim and coworkers synthesized the crosslinked styrene-acrylate copolymers and specifically investigated their swelling properties. The effect of the various polymerization conditions (for example, initiator concentration, polymerization temperature, crosslinker concentration) and the effect on moisture were studied through the oil absorption test using kerosene oil. Kim and coworkers concluded that oil absorbency is influenced mainly by the degree of the crosslinking and the hydrophobicity of the copolymer units; the copolymer with the longer acrylate chain displays higher oil absorbency. ${ }^{31,32}$

Recently, we synthesized crosslinked poly(orthocarbonate)s based on tetraethyl orthocarbonate for organic solvent absorption. ${ }^{33}$ All synthesized poly(orthocarbonate)s are thermally stable and have very fast and very good solvent uptake abilities. However, to the best of our knowledge, no other studies have reported the use of crosslinked 
poly(orthosilicate) as organic solvent absorbents. In our earlier paper, we suggested that crosslinked poly(orthosilicate)s can be synthesized by condensing tetraethyl orthosilicate (TEOS) and 1,4 cyclohexanedimethanol. ${ }^{34}$ This polymer is thermally stable and has a fast and good organic solvent uptake ability. As a result, crosslinked poly (orthosilicate)s can be used as absorbent material for organic solvents. Following the same strategy, we report in this study the synthesis, characterization and properties of new crosslinked poly(orthosilicate)s based on cyclohexanediol derivatives for organic solvent absorption.

\section{EXPERIMENTAL PROCEDURE}

\section{Materials}

The highest purity grade chemicals (cis-trans 1,3 cyclohexanediol, cis-trans 1,4 cyclohexanediol, trans 1,2 cyclohexanediol, TEOS, tetrahydrofuran (THF), benzene, dichloromethane and acetone) available from Aldrich (Taufkirchen bei München, Germany) were used without further purification.

\section{Characterization}

Fourier transform infrared spectroscopy (FT-IR) spectra were recorded on a Bio-Rad FTS 175C FT-IR spectrophotometer (Bio-Rad, Hercules, CA, USA) using $\mathrm{KBr}$ pellets. ${ }^{13} \mathrm{C}$ and ${ }^{29} \mathrm{Si}$ solid-state nuclear magnetic resonance (NMR) spectra were recorded on a 500-MHz Varian Inova spectrometer (Varian, Palo Alto, CA, USA) in a magic angle spinning (MAS) probe at $75.476 \mathrm{MHz}$. Thermogravimetric analysis (TGA) was performed under a nitrogen atmosphere at $10^{\circ} \mathrm{C} \mathrm{min}^{-1}$ using a Mettler Toledo model TGA/SDTA 851 (Mettler Toledo, Greifensee, Switzerland). Differential scanning calorimetry was performed with a Mettler TA Instrument DSC 822 at a heating rate of $10^{\circ} \mathrm{C} \mathrm{min}^{-1}$ under a nitrogen atmosphere.

\section{Polymer synthesis}

Different crosslinked polymers were synthesized using TEOS and cyclohexanediol derivative (mixture of cis-trans 1,3-cyclohexanediols, mixture of cis-trans 1,4-cyclohexanediol and trans 1,2-cyclohexanediol) monomers, as explained below (Scheme 1).

Synthesis of Poly 1. Using a Pyrex $(75 \mathrm{ml})$ pressure vessel (Chemglass, Vineland, NJ, USA) at $160^{\circ} \mathrm{C}$ in an oil bath, 1,3-cyclohexanediol $(0.75 \mathrm{~g}, 6.4 \mathrm{mmol})$ and TEOS $(0.72 \mathrm{ml}, 3.2 \mathrm{mmol})$ were allowed to react for 8 days. The resulting product was washed with water, ethanol and ether and vacuum dried to produce $0.45 \mathrm{~g}$ of a yellow-brown crosslinked solid polymer. FT-IR: 3420, 2940, 2863, 1057, 995, 926, $872 \mathrm{~cm}^{-1} \cdot{ }^{13} \mathrm{C}$ cross-polarization (CP)MAS NMR: 70.5, 59.4, 43.66, 35.09, 18.93 p.p.m. ${ }^{29}$ Si CPMAS NMR: $-62.48,-68.96$ p.p.m.

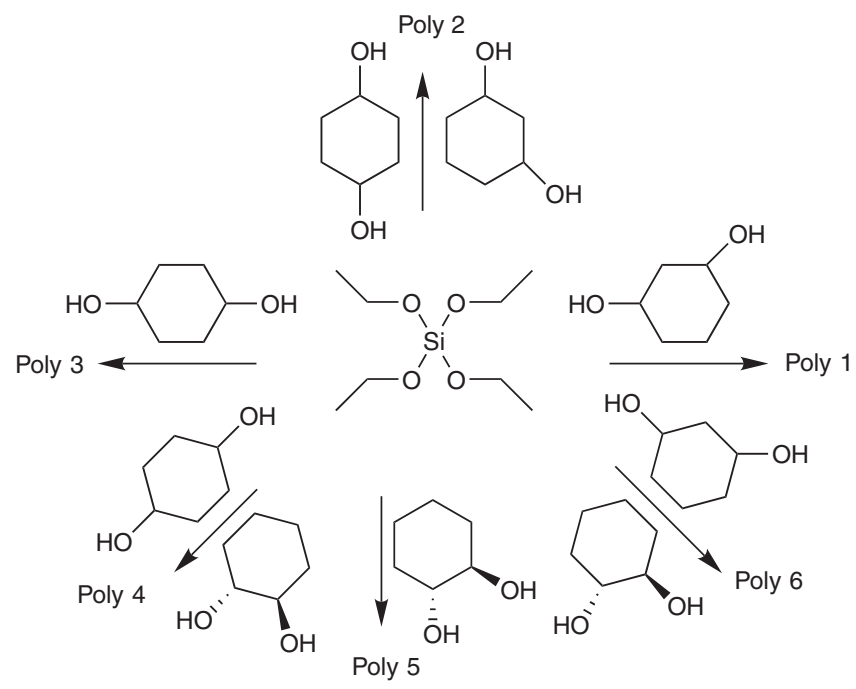

Scheme 1 Polymerization of cyclohexanediol derivatives with TEOS.
Anal. Calculated for $\mathrm{C}_{12} \mathrm{H}_{20} \mathrm{O}_{4} \mathrm{Si}$ : C, $56.25 \%$; H, 7.81\%. Found: C, $52.51 \%$; H, 7.77\%. Hydroxyl equivalent: 0.0214 .

Synthesis of Poly 2. The reaction of 1,3-cyclohexanediol (0.25 g, $2.1 \mathrm{mmol})$, 1,4-cyclohexanediol $(0.25 \mathrm{~g}, 2.1 \mathrm{mmol})$ and TEOS $(0.48 \mathrm{ml}, 2.1 \mathrm{mmol})$ at $160^{\circ} \mathrm{C}$ for 6 days in a pressure vessel. The resulting product was washed with water, ethanol and ether and vacuum dried to produce $0.33 \mathrm{~g}$ of a yellow-brown crosslinked solid polymer. FT-IR: $3431,2941,2863,1076,964,895 \mathrm{~cm}^{-1} \cdot{ }^{13} \mathrm{C}$ CPMAS NMR: 71.42, 33.89, 18.80 p.p.m. ${ }^{29}$ Si CPMAS NMR: $-58.78,-52.21$, -35.55 p.p.m. Anal. Calculated for $\mathrm{C}_{12} \mathrm{H}_{20} \mathrm{O}_{4} \mathrm{Si}$ : C, $56.25 \%$; H, $7.81 \%$. Found: C, $49.05 \%$; H, 7.82\%. Hydroxyl equivalent: 0.0206 .

Synthesis of Poly 3. The reaction of 1,4-cyclohexanediol (2.29 g, $19.7 \mathrm{mmol})$ and TEOS $(2.19 \mathrm{ml}, 9.85 \mathrm{mmol})$ at $180^{\circ} \mathrm{C}$ for 5 days in a pressure vessel generated a light brown polymer. The resulting product was washed with water, ethanol and ether and vacuum dried to produce $1.07 \mathrm{~g}$ of a crosslinked solid polymer. FT-IR: $3400,2943,2848,1063,966,867 \mathrm{~cm}^{-1} .{ }^{13} \mathrm{C}$ CPMAS NMR: 71.78, 59.68, 32.08, 18.40 p.p.m. ${ }^{29} \mathrm{Si}$ CPMAS NMR: $-74.90,-68.51$, -62.51 p.p.m. Anal. Calculated for $\mathrm{C}_{12} \mathrm{H}_{20} \mathrm{O}_{4} \mathrm{Si}$ : C, 56.25\%; H, 7.81\%. Found: C, $54.67 \%$; H, 8.15\%. Hydroxyl equivalent: 0.0286 .

Synthesis of Poly 4. 1,4-cyclohexanediol (3.9 g, $33.5 \mathrm{mmol})$, trans 1,2-cyclohexanediol $(3.9 \mathrm{~g}, 33.5 \mathrm{mmol})$ and TEOS $(7.48 \mathrm{ml}, 33.5 \mathrm{mmol})$ were polymerized in a pressure vessel at $160^{\circ} \mathrm{C}$. After 3 days, the polymerization was stopped, and a light brown crosslinked polymer was obtained. The resulting product was washed with water, ethanol and ether and vacuum dried to produce $5.48 \mathrm{~g}$ of polymer. FT-IR: $3400,2936,2862,1072,965,897 \mathrm{~cm}^{-1} \cdot{ }^{13} \mathrm{C}$ CPMAS NMR: 72, 59.30, 33.85, 18.63 p.p.m. ${ }^{29}$ Si CPMAS NMR: $-79.40,-67.88$, -60.94 p.p.m. Anal. Calculated for $\mathrm{C}_{12} \mathrm{H}_{20} \mathrm{O}_{4} \mathrm{Si}$ : C, $56.25 \%$; $\mathrm{H}, 7.81 \%$. Found: C, $42.95 \%, ; \mathrm{H}, 6.88 \%$. Hydroxyl equivalent: 0.0162 .

Synthesis of Poly 5. Trans 1,2-cyclohexanediol (2.57 g, $22.1 \mathrm{mmol})$ and TEOS $(2.46 \mathrm{ml}, 11.05 \mathrm{mmol})$ were placed in a pressure vessel at $160^{\circ} \mathrm{C}$ for 4 days to create a light brown crosslinked polymer. The resulting product was washed with water, ethanol and ether and vacuum dried to produce $1.04 \mathrm{~g}$ of polymer. FT-IR: 3600, 2955, 2880, 1080, $947 \mathrm{~cm}^{-1} .{ }^{13} \mathrm{C}$ CPMAS NMR: 67.20, 58.70, 22.49, 15 p.p.m. ${ }^{29} \mathrm{Si}$ CPMAS NMR: -110 p.p.m. Anal. Calculated for $\mathrm{C}_{12} \mathrm{H}_{20} \mathrm{O}_{4} \mathrm{Si}$ : C, 56.25\%; H, 7.81\%. Found: C, 44.61\%; H, 7.68\%. Hydroxyl equivalent: 0.0287 .

Synthesis of Poly 6. 1,3-cyclohexanediol (4.54 g, $39.07 \mathrm{mmol})$, trans 1,2-cyclohexanediol $(4.54 \mathrm{~g}, 39.07 \mathrm{mmol})$ and TEOS $(8.71 \mathrm{ml}, 39.07)$ were polymerized in a pressure vessel at $160^{\circ} \mathrm{C}$. After 3 days, the polymerization was stopped, and a yellow-brown crosslinked polymer was obtained. The resulting product was washed with water, ethanol and ether and vacuum dried to produce $4.0 \mathrm{~g}$ of polymer. FT-IR: $3400,2940,2864,1061,965 \mathrm{~cm}^{-1}$. ${ }^{13} \mathrm{C}$ CPMAS NMR: 67.20 , 35.32, 25.44 p.p.m. ${ }^{29} \mathrm{Si}$ CPMAS NMR: -110 p.p.m. Anal. Calculated for $\mathrm{C}_{12} \mathrm{H}_{20} \mathrm{O}_{4} \mathrm{Si}$ : C, $56.25 \%, \mathrm{H}, 7.81 \%$. Found: C, $28.22 \%, \mathrm{H}, 4.93 \%$. Hydroxyl equivalent: 0.0182 .

\section{Techniques}

Soluble fraction. A weighed quantity of crosslinked polymer was placed in a solvent, and the soluble fraction (SF) was extracted for 48 or $72 \mathrm{~h}$ using THF or dichloromethane. After extraction, the swelled polymers were vacuum dried at $45^{\circ} \mathrm{C}$. The SF was calculated using the following equation: ${ }^{35}$

$$
\mathrm{SF}(\%)=\frac{\left(W_{0}-W\right)}{W} \times 100
$$

where $W_{0}$ and $W$ are the weights of the polymers before and after extraction, respectively.

Swelling test. Bags made of filter paper were used to determine the swelling properties of the crosslinked polymers. ${ }^{36}$ First, the bags were immersed in the solvent and blotted quickly with an absorbent paper, followed by the addition of a dried polymer sample of known weight into the bags. The filled bags were immersed in the solvent. All the swelling experiments were conducted at room temperature. The equilibrium swelling values were determined based on the sample weights following immersion in an organic solvent for $24 \mathrm{~h}$. The bags were 
then removed, and their surfaces were blot dried gently and weighed in a stoppered weighing bottle. Solvent uptake percentages were calculated by the following formula:

$$
\text { Solvent uptake }(\%)=\frac{\left(W_{\mathrm{s}}-W_{\mathrm{d}}\right)}{W_{\mathrm{d}}} \times 100
$$

where $W_{\mathrm{d}}$ and $W_{\mathrm{s}}$ are the weights of the dry and swollen crosslinked polymer samples, respectively. For swelling measurements, $0.2 \mathrm{~g}$ of polymer sample was generally used.

Swelling kinetics. Measurements of swelling kinetics were conducted following the above-mentioned procedures using THF as a solvent. After removing the bags at various time intervals, they were quickly blot dried to remove THF attached to the surface and weighed.

Desorption kinetics. The THF retention of the polymers was determined by weighing the swollen polymer in air as a function of time.

\section{RESULTS AND DISCUSSION}

Synthesis and characterization of crosslinked poly(orthosilicate)s Different crosslinked poly(orthosilicate)s were synthesized using TEOS and cyclohexanediol derivatives (Scheme 1) by heating the reaction mixture at a moderately high temperature in a pressure vessel. To investigate the effect of using different cyclohexanediol derivatives on polymer properties and solvent absorption capacities, various crosslinked polymers were synthesized. In the synthesis of Poly 1, Poly 3 and Poly 5, one cyclohexanediol derivative monomer was condensed with TEOS, whereas for the synthesis of Poly 2, Poly 4 and Poly 6, a mixture of two cyclohexanediol monomers was used to synthesize the crosslinked polymers. Polymerization attempted with 1,4 cyclohexanediol and TEOS at $160^{\circ} \mathrm{C}$ did not produce a crosslinked polymer; only a highly viscous liquid was obtained. However, on increasing the temperature to $180^{\circ} \mathrm{C}$, the desired crosslinked polymer was obtained. All polymers were insoluble in organic solvents such as THF, dichloromethane, benzene and acetone, although they all displayed swelling abilities in these solvents, with the exception of Poly 3.

The structure of the resulting crosslinked polymers was investigated by FT-IR, solid-state CPMAS ${ }^{13} \mathrm{C}$ and ${ }^{29} \mathrm{Si} \mathrm{NMR}$, as well as thermal analysis. The strong stretching vibrations at 1057 and $872 \mathrm{~cm}^{-1}$ indicated the presence of $\mathrm{Si}-\mathrm{O}-\mathrm{C}$ and $\mathrm{Si}-\mathrm{O}-\mathrm{CH}$, respectively. Aliphatic absorptions were observed in the FT-IR spectra of the polymers at 2940 and $2863 \mathrm{~cm}^{-1}$. The IR spectrum also revealed a broad absorption peak between 3200 and $3600 \mathrm{~cm}^{-1}$, characteristic of the stretching vibrations of the hydroxyl group. This spectrum indicated that the desired structure contained the hydroxyl functionality as the end group, which is composed of the cyclohexanediol monomers. The elemental analyses were generally consistent with the theoretical values, except for Poly 6, which was found to be smaller than expected.

A solid-state ${ }^{13} \mathrm{C}$ CPMAS NMR spectrum of Poly $\mathbf{1}$ is presented in Figure 1 as an example. From the five major peaks at $\delta 70.5,59.4$, 43.66, 35.09 and 18.93 p.p.m., the second two resonances, 70.5 and 59.4 p.p.m., prove the presence of $-\mathrm{CH}-\mathrm{O}-\mathrm{Si}$ and $-\mathrm{CH}_{2}-\mathrm{O}$, respectively. The signals at 43.66 and 35.09 p.p.m. represent to the cyclohexane carbons, and the peak around 18.93 p.p.m. represents to the methyl end groups.

The solid-state ${ }^{29}$ Si CPMAS NMR spectrum of Poly $\mathbf{1}$ is shown in Figure 2 as an example. From the solid-state ${ }^{29} \mathrm{Si}$ CPMAS NMR result, the signals at -62.48 p.p.m. shows that the $\mathrm{Si}$ is bound to the hydroxyl group $(-\underline{\mathbf{S}}-\mathrm{OH})$ and at -68.96 p.p.m. refers to $-\mathrm{CH}-\mathrm{O}-\underline{\mathbf{S i}}$ group and reveal $\mathrm{Si}$ incorporated into the polymer structure. At $-110 \mathrm{p} . \mathrm{p} . \mathrm{m}$. there was an additional peak for Poly 5 and Poly 6 , indicative of the $-\underline{S i O}_{4}$ group.

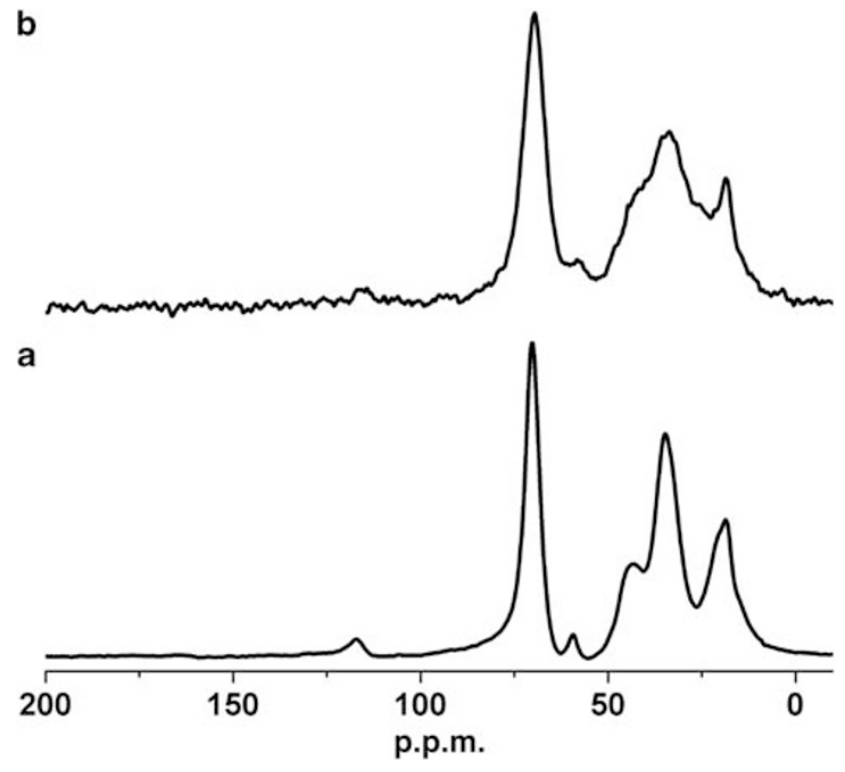

Figure 1 CPMAS solid-state ${ }^{13} \mathrm{C}$ NMR spectrum of Poly 1 before (a) and after (b) swelling measurements.

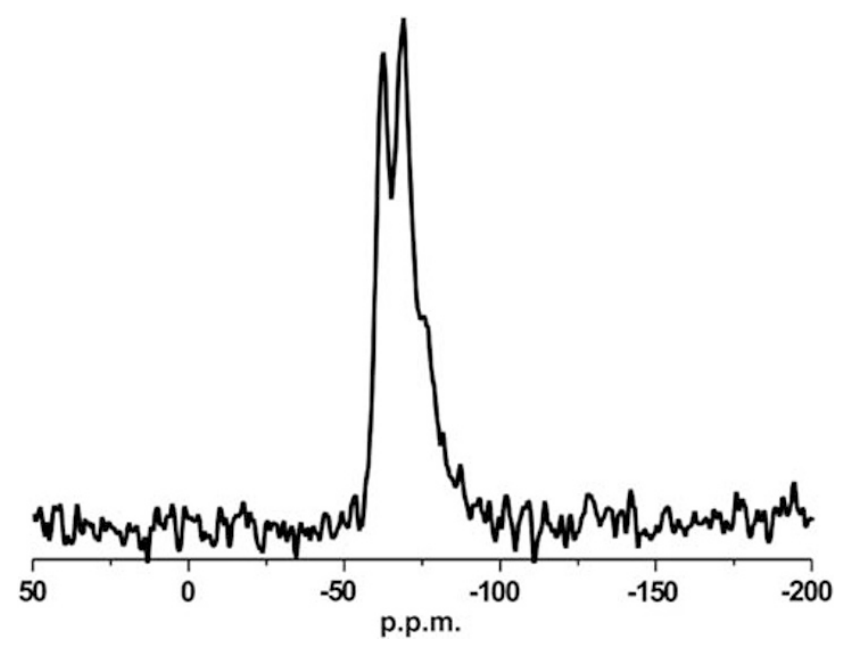

Figure 2 CPMAS solid-state ${ }^{29}$ Si NMR spectrum of Poly 1.

The thermal stability of the polymers was evaluated by TGA and differential scanning calorimetry under nitrogen. In the thermal analysis of all the polymers, neither the glass transition temperature $\left(T_{\mathrm{g}}\right)$ nor the melting point $\left(T_{\mathrm{m}}\right)$ was observed. The thermal stability of the polymers was identified to be in the following order: Poly $1 \approx$ Poly $3>$ Poly $6>$ Poly $4>$ Poly $5>$ Poly 2 . All the polymers exhibited thermal stability up to about $200^{\circ} \mathrm{C}$ with a $3 \%$ weight loss, except Poly 5 and Poly 2, which were slightly unstable when compared with the other polymers, showing a weight loss of 4 and $9 \%$ at the same temperature $\left(200^{\circ} \mathrm{C}\right)$, respectively (Figure 3$)$. The silicon content of the polymers was determined by TGA and found to be $27 \%$ for Poly 1 , $28.4 \%$ for Poly 3, 35\% for Poly 2, 42\% for Poly 4, 49\% for Poly 6 and 84\% for Poly 5 .

The remaining hydroxyl groups of the polymers were determined titrimetrically. The hydroxyl value was obtained from a sample of pyridine containing acetic anhydride that was first acetylized, after which the excessive acetylized reagent was hydrolized with water: this 


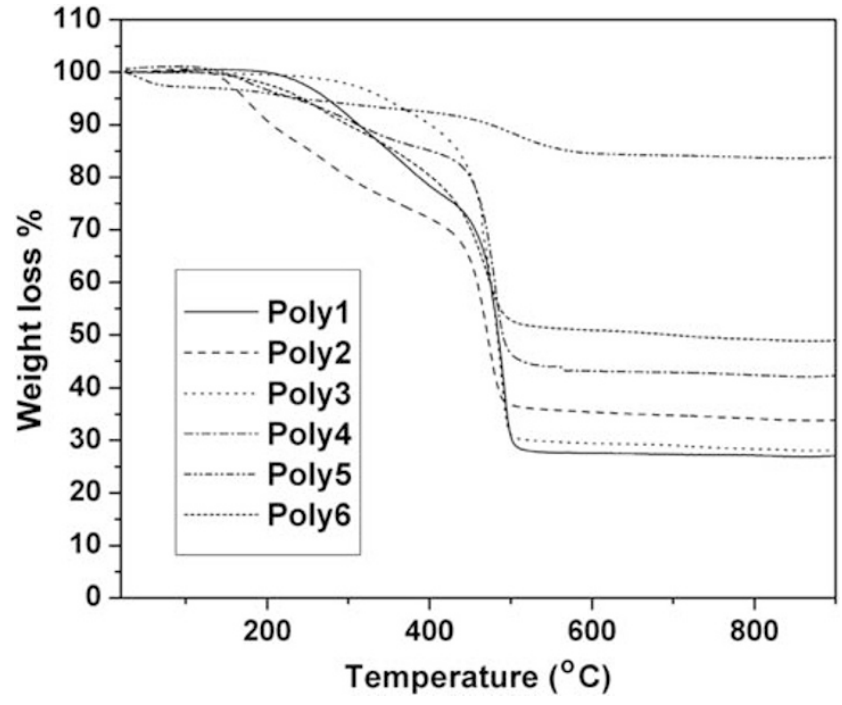

Figure 3 TGA results of crosslinked poly(orthosilicate)s.

generated acetic acid was titrated with sodium hydroxide to the end point. From this measurement, the hydroxyl equivalent of the polymers was found to be between 0.0162 and 0.0287 .

\section{Swelling properties of crosslinked poly(orthosilicate)s}

The synthesized crosslinked poly(orthosilicate)s were insoluble in THF, dichloromethane, acetone, benzene and other common organic solvents, although they possessed swelling abilities in these solvents, with the exception of Poly 3, which did not absorb these solvents.

Some polymer chains are not attached to the polymer network and can therefore be extracted from the gel fraction. These SFs can affect the swelling percentages of the polymers. Therefore, to minimize or eliminate this effect, the likely SFs in the crosslinked poly(orthosilicate)s were extracted using THF or dichloromethane. To achieve this, a known quantity of dried crosslinked polymer was added to the solvent, and the SFs were extracted for $48 \mathrm{~h}$. After extraction, the swelled polymers were vacuum dried at $45^{\circ} \mathrm{C}$. The SF values determined for the crosslinked polymers ranged between 5 and 38\%. The percentage of the extracted SFs depends on the type and concentration of the monomers and crosslinking agents. ${ }^{29}$ Poly 5, which was synthesized from the reaction of 1,2 cyclohexanediol and TEOS, had an SF value of $38 \%$. Using trans 1,2 cyclolehexanediol effectively blocked the crosslinking reaction; consequently, the SF value of Poly 5 was higher than for the other synthesized polymers.

The synthesized crosslinked poly(orthosilicate)s expressed good solvent uptake abilities in certain organic solvents such as tethrahydrofuran, benzene, dichloromethane and acetone. To measure the swelling after SF extraction, a known quantity of polymer sample was added to a bag and immersed in a solvent. All the swelling experiments were conducted at room temperature. After $24 \mathrm{~h}$, the bags were removed from the solvent, blotted quickly and weighed in a stoppered bottle. The calculated solvent uptake percentages of the polymers are shown in Table 1. Among the polymers, Poly $\mathbf{1}$ and Poly $\mathbf{2}$ showed the best results, and their maximum absorption capacity was seen in THF.

Poly 3, synthesized by the condensation of TEOS and 1,4 cyclohexanediol, showed no swelling in the organic solvent because of its rigid rod-like structure; this polymer had high thermal stability. In contrast, when the TEOS was condensed with 1,4 cyclohexanediol along with 1,3 cyclohexanediol (Poly 2), the resulting polymer showed good
Table 1 Swelling properties of crosslinked poly(orthosilicate)s

\begin{tabular}{lrrrrr}
\hline Solvents & Poly $\mathbf{1}$ & Poly $\mathbf{2}$ & Poly $\mathbf{4}$ & Poly $\mathbf{5}$ & Poly 6 \\
\hline THF & 155 & 165 & 116 & 100 & 115 \\
Dichloromethane & 108 & 121 & 110 & 98 & 115 \\
Benzene & 85 & 114 & 80 & 87 & 84 \\
Acetone & 85 & 68 & 73 & 72 & 76 \\
\hline
\end{tabular}

Abbreviation: THF, tetrahydrofuran.

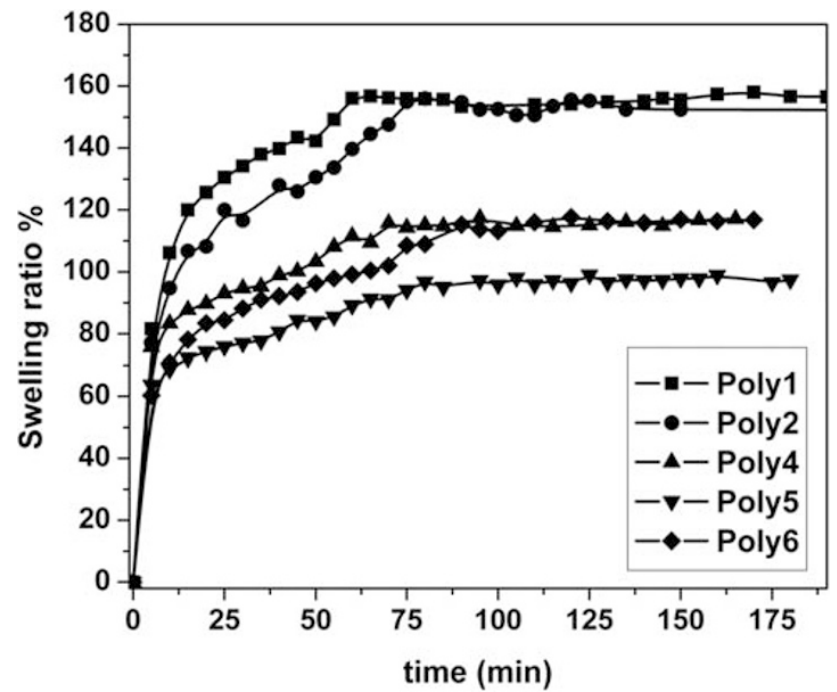

Figure 4 Swelling kinetics of crosslinked poly(orthosilicate)s in THF at room temperature. Each point presented in the figure is an average of at least four different measurements. There is a maximum $5 \%$ deviation from each point.

absorption ability. These high swelling percentages can be explained by addition of the cis-trans 1,3 cyclohexanediol monomer in a polymer structure, which gives the polymer more flexibility, enabling the solvent to easily penetrate into the chains. When comparing the swelling percentages of Poly $\mathbf{1}$ and Poly 6, the latter reveals a less significant swelling uptake ability than the former. Using trans 1,2 cyclohexanediol in polymer synthesis minimizes the voids within the polymeric network, and thus less solvent can penetrate into this space.

In Figure 4, the swelling kinetics of the polymers in THF is given. To evaluate the saturation time of the crosslinked poly(orthosilicate)s, the swelling percentages of polymers with time were examined. All the polymers had very fast solvent uptake capabilities. For example, Poly 1 reached $82 \%$ swelling in $5 \mathrm{~min}$ and reached saturation at $60 \mathrm{~min}$.

All the crosslinked poly(orthosilicate)s released the absorbed THF very quickly (Figure 5). The THF retention of polymers was followed in time by examining the weight loss of the swollen polymer in air. Within an average of 20-25 min, all the polymers released almost all the absorbed THF. All the synthesized crosslinked poly(orthosilicate)s based on cyclohexanediol achieved the original swollen state without any capacity loss. These results clearly indicate that all the polymers are easily regenerable. Following regeneration, examination by FT-IR and NMR was performed to see whether there were any changes because of further condensation reactions by unreacted groups or hydrolyzation of Si-O-C bonds. Figure 1 shows the ${ }^{13} \mathrm{C}$ NMR spectra of the Poly 1 before and after swelling (four times swelling/deswelling) measurements. These results revealed no change in the FT-IR and 


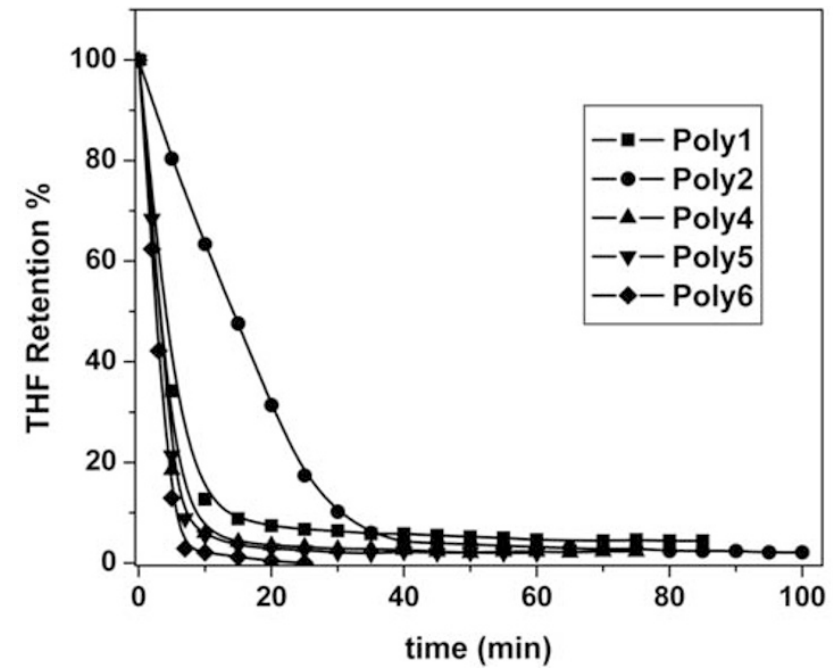

Figure 5 THF retention of crosslinked poly(orthosilicate)s in air. Each point presented in the figure is an average of at least four different measurements. There is a maximum $5 \%$ deviation from each point.

NMR spectra of the polymers, and the synthesized crosslinked polyorthosilicates were quite stable for repeated measurements.

\section{CONCLUSIONS}

In conclusion, we report a simple synthesis of novel crosslinked poly(orthosilicate)s and their use as organic solvent absorbents. All these polymers are moderately thermally stable and possess very fast and good solvent uptake abilities. The absorbed solvent can be easily removed from the polymers; thus, the crosslinked poly(orthosilicate)s can be used several times without any loss of absorption capacity.

\section{ACKNOWLEDGEMENTS}

We thank the Scientific and Technological Research Council of Turkey-TUBITAK for the support of this work through Grant TBAG/106T616.

1 Davis, S. C., Hellens, W. V. \& Zahalka, H. A. Polymeric Materials Encyclopedia (ed. Salamone, J.C.) Vol. 4, 2264 (CRC Press, Rochester, NY, 1996).

2 Bertrand, P. A. Oil absorption into cotton-phenolic material. J. Mater. Res. 8, 1749-1757 (1993).

3 Zhang, Y. Q., Tanaka, T. \& Shibayama, M. Super-absorbency and phase transition of gels in physiological salt solutions. Nature 360, 142-144 (1992).

4 Alexandratos, S. D. \& Natesan, S. Ion-selective polymer-supported reagents: the principle of bifunctionality. Eur. Polym. J. 35, 431-436 (1999).

5 Stegmann, R., Lotter, S., King, L. \& Hopping, W. D. Fate of an absorbent gelling material for hygiene paper products in landfill and composting. Waste Manag. Res. 11, 155-170 (1993).

6 Hosoya, K., Kageyama, Y., Kimata, K., Araki, T., Tanaka, T. \& Frechet, J. M. J. Preparations and properties of uniform size macroporous polymer beads prepared by two-step swelling and polymerization method utilizing divinyl succinate or divinyl adipate as a crosslinking agent. J. Polym. Sci. Polym. Chem. 34, 2767-2774 (1996).

7 Dagani, R. Molecular magic with microwaves. Chem. Eng. News. 10, 26-33 (1997).

8 Doyle, F. J., Dorski, C., Harting, J. E. \& Peppas, N. A. Control and modeling of drug delivery devices for the treatment of diabetes. Proc. 1995 Am. Control Conf. 1, 776-780 (1995).

9 Osada, Y., Gong, J. P. \& Tanaka, T. Polymer gels. J. Macromol. Sci. C Polym. Rev. C44, 87-112 (2004).

10 Ricka, J. \& Tanaka, T. Swelling of ionic gels: quantitative performance of the Donan theory. Macromolecules 17, 2916-2921 (1984).

11 llavsky, M. Phase transition in swollen gels. 2. Effect of charge concentration on the collapse and mechanical behavior of polyacrylamide networks. Macromolecules 15, 782-788 (1982).

12 Bajpai, S. K. Swelling-deswelling behavior of poly(acrylamide-co-maleic acid) hydrogels. J. Appl. Polym. Sci. 80, 2782-2789 (2001).

13 Annaka, M. \& Tanaka, T. Multiple phases of polymer gels. Nature 355, 430-432 (1992).

14 Hirokawa, Y. \& Tanaka, T. Volume phase transition in a nonionic gel. J. Chem. Phys. 81, 6379-6380 (1984).

15 Siegel, R. A. \& Firestone, B. A. pH-dependent equilibrium swelling properties of hydrophobic polyelectrolyte copolymer gels. Macromolecules 21, 3254-3259 (1988).

16 Langer, R. \& Tirrell, D. A. Designing materials for biology and medicine. Nature 428, 487-492 (2004).

17 Kim, J., Nayak, S. \& Lyon, L. A. Bioresponsive hydrogel microlenses. J. Am. Chem. Soc. 127, 9588-9592 (2005).

18 Peppas, N. A. \& Langer, R. New challenges in biomaterials. Science 263, 1715-1720 (1994).

19 Park, T. G. Temperature modulated protein release from $\mathrm{pH} /$ temperature sensitive hydrogels. Biomaterials 20, 517-521 (1999).

20 Jeong, B., Bae, Y., Lee, H. D. S. \& Kim, S. W. Biodegradable block copolymers as injectable drug-delivery systems. Nature 388, 860-862 (1997).

21 Hoffman, A. S. Applications of thermally reversible polymers and hydrogels in therapeutics and diagnostics. J. Control. Release 6, 297-305 (1987).

22 Holtz, J. H. \& Asher, S. A. Polymerized colloidal crystal hydrogel films as intelligent chemical sensing materials. Nature 389, 829-832 (1997).

23 Atta, A. M. Swelling behaviors of polyelectrolyte hydrogels containing sulfonate groups. Polym. Adv. Technol. 13, 567-576 (2002).

24 Zhou, M. H. \& Cho, W. J. Oil absorbents based on styrene-butadiene rubber. J. Appl. Polym. Sci. 89, 1818-1824 (2003).

25 Champ, S., Xue, W. \& Huglin, M. B. A novel semi-automated apparatus to concentrate aqueous polymer solutions with a thermosensitive hydrogel. Polymer 42, 6439-6445 (2001).

26 Zhou, M. H., Kim, S. H., Park, J. G., Ha, C. S. \& Cho, W. J. Preparation and oilabsorptivity of crosslinked polymers containing stearylmethacrylate, 4-t-butylstyrene, and divinylbenzene. Polym. Bull. 44, 17-24 (2000).

27 Martel, B. \& Morcellet, M. Sorption of aromatic compounds in water using polymer sorbents containing amino groups. J. Appl. Polym. Sci. 51, 443-451 (1994).

28 Zhou, M. H. \& Cho, W. J. High oil-absorptive composites based on 4-tert-butylstyreneEPDM-divinylbenzene graft polymer. Polym. Int. 50, 1193-1200 (2001).

29 Flory, P. J. Principles of Polymer Chemistry. Ch. 13, 541-593 (Cornell University Press, Ithaca, NY, 1953).

30 Zhang, G. Q., Zhou, M. H., Ma, J. H. \& Liang, B. R. Preparation and swelling properties of solution crosslinked poly(cis-1,4-butadiene) gels. J. Appl. Polym. Sci. $90,2241-2245$ (2003).

31 Jang, J. \& Kim, B. Studies of crosslinked styrene-alkyl acrylate copolymers for oil absorbency application. I. Synthesis and characterization. J. Appl. Polym. Sci. 77, 903-913 (2000).

32 Jang, J. \& Kim, B. Studies of crosslinked styrene-alkyl acrylate copolymers for oil absorbency application. II. Effects of polymerization conditions on oil absorbency. J. Appl. Polym. Sci. 77, 914-920 (2000).

33 Sonmez, H. B. \& Wudl, F. Cross-linked poly(orthocarbonate)s as organic solvent sorbents. Macromolecules 38, 1623-1626 (2005).

34 Sonmez, H. B., Karadag, K. \& Onaran, G. Use of crosslinked poly(orthosilicate)s as organic solvent absorbent. Polym. Prep. Am. Chem. Soc. 49, 846 (2008).

35 Atta, A. M. \& Arndt, K. F. Synthesis of charged linear and crosslinked maleic diester polymers with electron-beam irradiation. Polym. Int. 52, 389-398 (2003).

36 Acar, N. Radiation synthesis of poly(2-vinylpyridine) gels and their swelling characteristics. Rad. Phys. and Chem. 63, 185-191 (2002). 